\title{
Research on Motivation Coupling Relationship of Various People Participation in Taijiquan Based on Principal Component Method and Matrix Theory
}

\author{
Guo Li \\ School of Physical Education and Health Sciences, Bijie University, Bijie, China \\ I_i_g_uo@126.com
}

Keywords: principal component analysis; study motivation; coupling relationship; matrix theory; intrinsic motivation; development strategy

\begin{abstract}
Every people needs an internal power when participate in a thing or a movement. The intrinsic power is the motivation. Now, there are more and more people to practice taiji and the ages are more and more young. The paper analyzes practice motivation of practitioners in different ages according to principal component analysis and researches the influence of taijiquan on the physical and mental development of practitioners. Finally, this paper confirms the irreplaceable positive role of taijiquan's movable orderly and neat for the body exercise and psychological stress free of any practitioner. This paper forecasts and analyzes the development strategy of taijiquan and encourages more and more people participate and insist in taijiquan, and so as to improve their immunity and happy degrees.
\end{abstract}

\section{Introduction}

With the fast development of the socialist market economy, and people's life is speed up. In such a high speed rotating, peoples of sub-health are more and more young. Tired and high pressures are along with more and more people in every day. So, the slow gentle movement-taijiquan also highly praised by more and more people[1]. Persons of different ages began to learn and practice taijiquan for various reasons and purposes. For this kind of national fitness and popular phenomenon of taijiquan, many researchers and experts of health and social have done many investigations, and do the further analysis of the generation of this kind of phenomenon. For example, Lin Wang analyzes the human physiological health, mental health, life philosophy and religion and finds that the practice of taijiquan has correct and positive influence on self-perception of college students and at the same time, she concludes that the traditional culture has irreplaceable positive role on students' values, world outlook education of higher education. Zhijun Li, Xunzhang Shen, Enqian Ruan, Jianqing Zhuang investigate and analyze the present situation of elderly practice taijiquan by questionnaire. Through the survey, they find that old people practice taijiquan is mostly in order to strengthen the body and to prevent and cure diseases. This slow motion--taijiquan is especially suitable for old people whose body function is gradually decline. Their research also shows that practicing taijiquan is great help to old people's physical and mental health. Baoping Song studies attitude and practice motivation of Shanghai university students to practice taijiquan and karate by using information collection, literature study and questionnaire methods[2,3]. Finally, he analyzes the relativity and independence of taijiquan and karate and finds that the development and maintenance of the movement has a great impetus on the promotion of teenager's body's development.

\section{Analysis on the impact of practicing taijiquan on body and mind}

\section{A. Cognitive influence of Taijiquan on body function}

Taijiquan embodies the artistic combination of dialectical thinking of Chinese nation and martial arts and also is the high-level performance of human culture. Taijiquan derived from ancient Taoism,which advocated movable orderly and couple hardness with softness and its action is like floating clouds and flowing water. Practitioners in the elegant movement sports can not only feel the 
rhythm of the music but also understand the connotation of philosophy. So, it is called "the quintessence of a country"[4]. Taijiquan which is slow and gentle movement adjusts breathing to smooth through sports. Microcirculation expansion can make the person body material and energy exchange with each other, so as to improve the body internal circulation, achieve physical fitness and prevent and cure diseases.

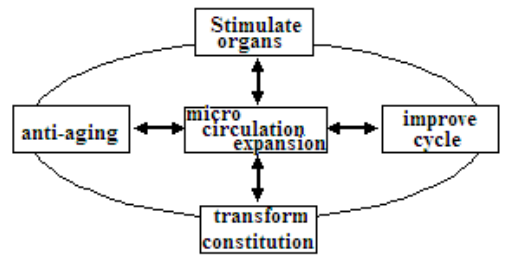

Figure 1. The cycle improvement plan of taijiquan's regimen on physical

According to the Figure 1, the traditional method of taijiquan advocates using the qigong and neixinggong to adjust the body microcirculation. It inspires and promotes body inner potential by certain intensity stimulus of tissues and organs and causes a series of adaptive change of the body's function. The direction of physique improvement towards the direction of enhancement, and the structure and function of all tissues and organs of body are also delay degenerative change follows the physical change [5]. This series of change in the body can effectively strengthen the body and delay senility.

B. The cognition of relevance of mental health and taijiquan

As an organic whole, the mind and body function of people are inseparable. In the medicine, we believed that taijiquan which is slow light emphasize maintain the condition of physical and mental pleasure and relaxation. This kind of light, loose and soft movement is more easily to the state of the union of the body and the spirit for practitioners[6]. Zero pressure state can block the inverse random interference effect of stimulated and fiery feelings on blood, and protect the coordination function of thinking consciousness on body inner circulation. Taijiquan stressed the transcendental attitude which not only can make a person feel peace of mind but also can effectively relieve the tremendous pressure of the modern life to people's psychological. Psychology thinks that the maintenance of cheerful and soothe emotions of body and minds on the one hand can help to philosophize, and ease the contradiction of life, and accept setbacks, on the other hand, can improve the psychological characteristics and perfect personality.

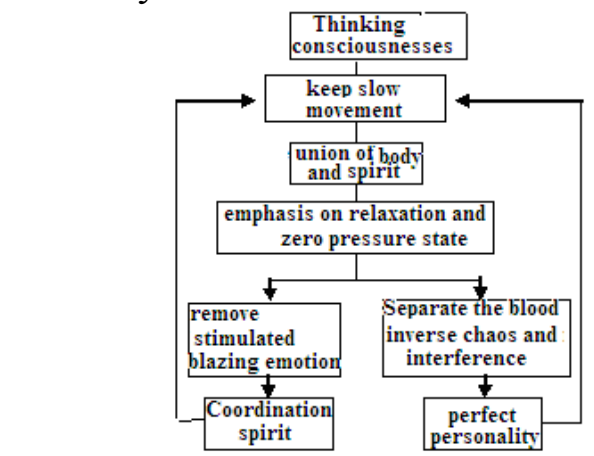

Figure 2. Analysis of relevance of taijiquan and mental health

From Figure 2, we can see that taijiquan comes from theory of yin-yang which mainly emphasis on a balance of Yin and Yang, harmony state. People come from taiji. The body and consciousness occupy two levels of Yin and Yang. The combination of dynamic and static is as the coordination of Yin and Yang. When they combined and tolerated with each other, the person state of mind is clear and people are happy and prolong life.

\section{Analyses on taijiquan exercise's influence factors on all kinds of people}

Research on the motivation of college students who practice taijiquan

Taijichuan is a strong Chinese traditional culture. Taiji's Yin and Yang theory emphasizes a state of balance which influenced by the Chinese traditional philosophy. In the personalized teaching mode, college students can independently choose the course of study. This research analyzes motivation of college students who learning taijiquan through the principal component analysis. For 
a motivation research material, we can observe $p$ motivation variables $x_{1}, x_{2}, \cdots x_{p}$. Data array of $n$ samples are shown as follows:

In this formula,

$$
X=\left(\begin{array}{cccc}
x_{11} & x_{12} & \cdots & x_{1 p} \\
x_{21} & x_{22} & \cdots & x_{2 p} \\
\vdots & \vdots & \vdots & \vdots \\
x_{n 1} & x_{n 2} & \cdots & x_{n p}
\end{array}\right)=\left(x_{1}, x_{2}, \cdots x_{p}\right)
$$

$$
x_{j}=\left(\begin{array}{c}
x_{1 j} \\
x_{2 j} \\
\vdots \\
x_{n j}
\end{array}\right), \quad j=1,2, \cdots p
$$

$p_{\text {new variables (variables) synthesized by }} p$ observation variables are as follows[7]:

$$
\left\{\begin{array}{c}
F_{1}=a_{11} x_{1}+a_{12} x_{2}+\cdots+a_{1 p} x_{p} \\
F_{2}=a_{21} x_{1}+a_{22} x_{2}+\cdots+a_{2 p} x_{p} \\
\cdots \\
F_{p}=a_{p 1} x_{1}+a_{p 2} x_{2}+\cdots+a_{p p} x_{p}
\end{array}\right.
$$

It can be short for:

$$
F_{j}=\alpha_{j 1} x_{1}+\alpha_{j 2} x_{2}+\cdots+\alpha_{j p} x_{p} \quad j=1,2, \cdots, p
$$

College students' practice motivation should meet the following requirements:

(1) $F_{i}, F_{j}$ are uncorrelated $(i \neq j, i, j=1,2, \cdots, p)$;

(2) variance of $F_{1}$ is greater than $F_{2}$ 's and $F_{3}$ 's. And we can analogize it like the following formula[8]:

$$
a_{k 1}^{2}+a_{k 2}^{2}+\cdots+a_{k p}^{2}=1 \quad k=1,2, \cdots p .
$$

So, we can call $F_{1}$ as the first principal component, call $F_{2}$ as the second principal component. We can get the ${ }^{p}$-th principal component by analogy. We call $a_{i j}$ as principal component factor. The model can be expressed by matrix as[9]: $F=A X$.

And,

$$
F=\left(\begin{array}{c}
F_{1} \\
F_{2} \\
\vdots \\
F_{p}
\end{array}\right) \quad X=\left(\begin{array}{c}
x_{1} \\
x_{2} \\
\vdots \\
x_{p}
\end{array}\right) \quad A=\left(\begin{array}{cccc}
a_{11} & a_{12} & \cdots & a_{1 p} \\
a_{21} & a_{22} & \cdots & a_{2 p} \\
\vdots & \vdots & \vdots & \vdots \\
a_{p 1} & a_{p 2} & \cdots & a_{p p}
\end{array}\right)=\left(\begin{array}{c}
a_{1} \\
a_{2} \\
\vdots \\
a_{p}
\end{array}\right)
$$

$A$ is principal component coefficient matrix.

From the matrix analysis, we can know that to practice taijiquan can exercise overall coordination of body and can exercise the outbreak ability of local muscle. When we move slowly, muscle keeps in the state of static force application which can exercise the endurance of muscle more than physical explosive movement. Flowing movement form of taijiquan can repeatedly convert the load and the centre of gravity of the whole body between two legs[10]. The slow practice method can overcome two leg muscle internal resistance through the circulation. Muscles and joints shrink and rotate in more than one angle and complete static practice. College students can obtain coordination body function and status, and keep the strong energy and body young state through the practice of taijiquan. It also can adjust learning mentality in a dull life of learning, and overcome the pressure 
brought by a heavy workload, and keep to themselves and perfect personality in the basis of rational thinking.

motivation analysis of old people to practice taijiquan

The bodies' functions of elders' are decline. With the growth of the age, there is something wrong about nervous system, cardiovascular system and digestive drainage system. Assuming that $p$ characteristic roots of correlation coefficient matrix $R$ are $\lambda_{1} \geq \lambda_{2} \geq \cdots \geq \lambda_{p}$. eigenvector is $a_{j}$.

$$
A=\left(\begin{array}{cccc}
a_{11} & a_{12} & \cdots & a_{1 p} \\
a_{21} & a_{22} & \cdots & a_{2 p} \\
\vdots & \vdots & \vdots & \vdots \\
a_{p 1} & a_{p 2} & \cdots & a_{p p}
\end{array}\right)=\left(\begin{array}{c}
a_{1} \\
a_{2} \\
\vdots \\
a_{p}
\end{array}\right)
$$

The variance of $F_{1}$ is:

$$
\operatorname{Var}\left(F_{1}\right)=a_{1} X X^{\prime} a_{1}^{\prime}=a_{1} R a_{1}^{\prime}=\lambda_{1}
$$

And, $\operatorname{Var}\left(F_{i}\right)=\lambda_{i}$. The variance of main components is decrease successively. The covariance is as follows.

$$
\operatorname{Cov}\left(a_{i}^{\prime} X^{\prime}, a_{j} X\right)=a_{i}^{\prime} R a_{j} \quad=a_{i}^{\prime}\left(\sum_{\alpha=1}^{p} \lambda_{\alpha} a_{\alpha} a_{\alpha}^{\prime}\right) a_{j} \quad=\sum_{\alpha=1}^{p} \lambda_{\alpha}\left(a_{i}^{\prime} a_{\alpha}\right)\left(a_{\alpha}^{\prime} a_{j}\right)=0, \quad i \neq j
$$

According to a proof, the principal component covariance of principal component analysis is diagonal matrix, the diagonal element of it is the characteristic value of the original data correlation matrices. The elements of principal component coefficient matrix $A$ are feature vector of original data correlation matrix Eigen values. Matrix $A$ is a orthogonal matrix.

So, new variables can be transformed from $\left(x_{1}, x_{2}, \cdots x_{p}\right)$.

$$
\left\{\begin{array}{c}
F_{1}=a_{11} x_{1}+a_{12} x_{2}+\cdots+a_{1 p} x_{p} \\
F_{2}=a_{21} x_{1}+a_{22} x_{2}+\cdots+a_{2 p} x_{p} \\
\cdots \\
F_{p}=a_{p 1} x_{1}+a_{p 2} x_{2}+\cdots+a_{p p} x_{p}
\end{array}\right.
$$

New random variables are unrelated with each other, and variances decrease in turn. The above variables analysis confirmed that the heart flash dynamic of taijiquan can maintain the balance of the body which finished in the nervous activity of brain. So, it trained the central nervous system of brain, and improved the sensitivity of the brain and central nerve. Taijiquan can lower diastolic blood pressure, improve contraction ability of cardiac. It is advantage to the blood discharge, and it can reduce the incidence of cardiovascular disease. Keep practicing taijiquan can delay the caducity of elderly nervous system and digestive drainage system, and reduce the incidence of disease, increase bone mineral density and resist hyperglycemia, improve the antioxidant ability, achieve a strong body and mind and prolong life role. All of this is the motives of old people who choose taijiquan.

\section{The development strategy of taijiquan}

The above analysis confirmed that taijiquan has the positive role on physical and mental health development of all peoples. We should encourage more and more people understand and try to practice taijiquan and carry forward the Chinese traditional culture in the process of body building. This study analyzes the current situation of the development of taijiquan and summarizes the development strategies which are shown as follows.

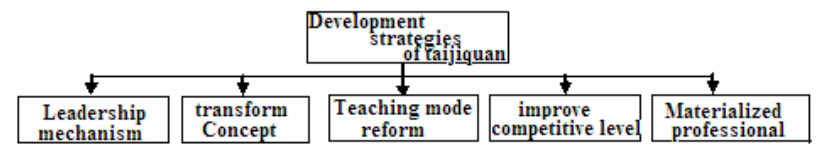

Figure 3. Future development strategy analysis of taijiquan 
According to Figure 3, we can know that the development of Taijichuan has been based on the folk spontaneous organizations which have no leadership and the technical abilities of members are uneven and it has no advantage on the long development of taijiquan. So, the government should organize the leadership mechanisms. Although taijiquan exercise is beneficial to the health of body and mind development, the knowledge of general public is little. So we should strengthen the propaganda of taijiquan, and change the old ideas, and promote taijiquan using film, television, network, magazines and other publicity channels. The traditional teaching mode of one-to-one can't meet the learning needs of the mass people, so we should also reform the traditional teaching mode of taijiquan. Arena contests and competitive sports can always arouse the learning enthusiasm of the people. To improve the competitive level is beneficial for the development of taijiquan. We should make the development of taijiquan substantiation and professional and carry forward the traditional culture in promoting economic development at the same time. When it comes to the development strategy of comprehensive, this is a win-win development strategy.

\section{Conclusion}

The above analysis confirmed that tiajiquan has positive role for the physical and mental health development of all peoples. College students can obtain coordination body function and status and keep the strong and young of the body through taijiquan. Practicing taijiquan can delay the caducity of elderly nervous system and digestive drainage system and reduce the incidence of disease. The study also proved that developing and maintaining the motivation of movement has a positive influence on body and mind development of youths and elders.

\section{References}

[1] Lin Wang. The attitude and influencing factors research of taijiquan exercise of college students .Beijing sports university, 2010: 21-23.

[2] Zhijun Li, Xunzhang Shen, Enqian Ruan, Jianqing Zhuang. Investigation and analysis of old people practicing in Shanghai community. Journal of Shanghai institute of physical education, 2013 (01):34-35.

[3] Baoping Song. The comparative study of motivation of taijiquan and karate of Shanghai college students . Science, fight martial arts,2008 (01):33-34.

[4] Xiaojun Feng. The present situation and countermeasures of taijiquan in China . Journal of Guangdong Education College,2009 (01):34-36.

[5] Shaojie Zhao. The investigation and analysis of the motivations of learning taijiquan in Zhengyou taijiquan class of Taipei College. New world, 2012(04):2-3.

[6] Lizhi Sun. The present situation investigation and countermeasures research of normal college students' motivation to learn taijiquan. East China normal university,2010:45-46.

[7] Yi Ke. The present situation investigation and analysis of old people practicing taijiquan in Wuhan. Wuhan institute of physical education, 2008: 43-44.

[8] Wei Shu. The consumption behavior status comparative study on taijiquan and the yoga in Beijing. Capital institute of physical education, 2009(03):45-46.

[9] Songbo Zheng. The taijiquan fitness principle. Jiangxi normal university, 2012:39-42.

[10] BangSheng Yin. Taijiquan development strategy in Hunan urban community. Hunan normal university ,2011:39-40. 